\title{
РАЗВИТИЕ ИССЛЕДОВАНИЙ ТЕПЛОВЫХ ПРОЦЕССОВ В ТЕХНОЛОГИИ МАШИНОСТРОЕНИЯ
}

В.Ф.БЕЗЬЯЗЫЧНЫЙ ${ }^{1}$, М.СЧЕРЕК ${ }^{2}$

${ }_{1}^{1}$ Рыбинский государственньй авиационный технологический университет имени П.А.Соловьева, Рыбинск, Россия

${ }^{2}$ Институт экологически рациональных технологий Национального научно-исследовательского института, Радом, Польша

\begin{abstract}
Изложена методика определения температуры в поверхностном слое обрабатываемой детали лезвийным инструментом с учетом объемного источника тепла в зоне резания, на основе которой предлагается расчетом определять погрешности обработки, обусловленные тепловым воздействием на режущий инструмент и обрабатываемую заготовку. При определении теплового воздействия на режущую часть инструмента учитываются тепловые потоки, действующие по передней и задней поверхности. При определении теплового воздействия на обрабатываемую деталь учитываются тепловые потоки, действующие от деформации материала при удалении стружки и задней поверхности режущего инструмента. Температура в зоне резания определяется суммированием температуры в поверхностном слое, возникающей от пластических деформаций материала в зоне резания, трения стружки о переднюю поверхность режущего инструмента и трения задней поверхности режущего инструмента об обработанную поверхность. Особенность предлагаемого метода состоит в том, что учитываются физико-механические свойства обрабатываемого и инструментального материалов (температуропроводность, предел прочности теплопроводность, удельная объемная теплоемкость), режимы обработки (скорость резания, подача и глубина резания), размеры обрабатываемой заготовки и режущего инструмента, геометрия режущей части инструмента (передний и задний углы, радиус при вершине резца в плане, радиус округления режущей инструмента, главный и вспомогательный углы в плане). В расчетах учитывается изменение интенсивности объемных тепловых потоков в зоне резания по их высоте.
\end{abstract}

Ключевые слова: обработка резанием; тепловые потоки в зоне резания; температура в зоне резания; погрешность обработки; режущий инструмент; обрабатываемая заготовка

Как цитировать эту статью: Безъязычный В.Ф. Развитие исследований тепловых процессов в технологии машиностроения / В.Ф.Безъязычный, М.Счерек // Записки Горного института. 2018. Т. 232. С. 395-400. DOI: 10.31897/PMI.2018.4.395

Введение. Рассматривая вопрос об изучении тепловых явлений в зоне резания, необходимо обратиться к работам выдающихся ученых в области технологии машиностроения, изучавших влияние тепловых явлений на точность обработки и качество поверхностного слоя деталей. Это прежде всего профессора А.Н.Резников, А.Д.Макаров, С.С.Силин, Н.В.Талантов, М.Ф.Полетика, В.В.Максаров [2, 3, 10], а также зарубежные авторы [8, 9, 11 и др.]. Наиболее подробно изложена схема действия тепловых источников в работе А.Н.Резникова (рис.1) [5], но расчетное определение их влияния на погрешности обработки изучено недостаточно.

В зоне резания действуют следующие тепловые потоки:

$$
\begin{gathered}
Q_{\text {и }}=Q_{\text {ди }}+Q_{\text {тз }}-Q_{3}, \\
Q_{\mathrm{c}}=Q_{\text {дс }}+Q_{\text {тп }}-Q_{\text {п }}, \\
Q_{\mathrm{p}}=Q_{\Pi}+Q_{3},
\end{gathered}
$$

где $Q_{\text {ди }}$ - часть теплоты деформации, уходящей в изделие; $Q_{\text {тз }}$ - часть теплоты, возникающей в результате трения между изделием и резцом; $Q_{3}$ - часть теплоты, возникающая в результате теплообмена на площадке контакта поверхности резания с задней поверхностью инструмента; $Q_{\text {дс }}-$ часть теплоты деформации, уходящей в стружку; $Q_{\text {п }}$ - часть теплоты, возникающая в резуль-

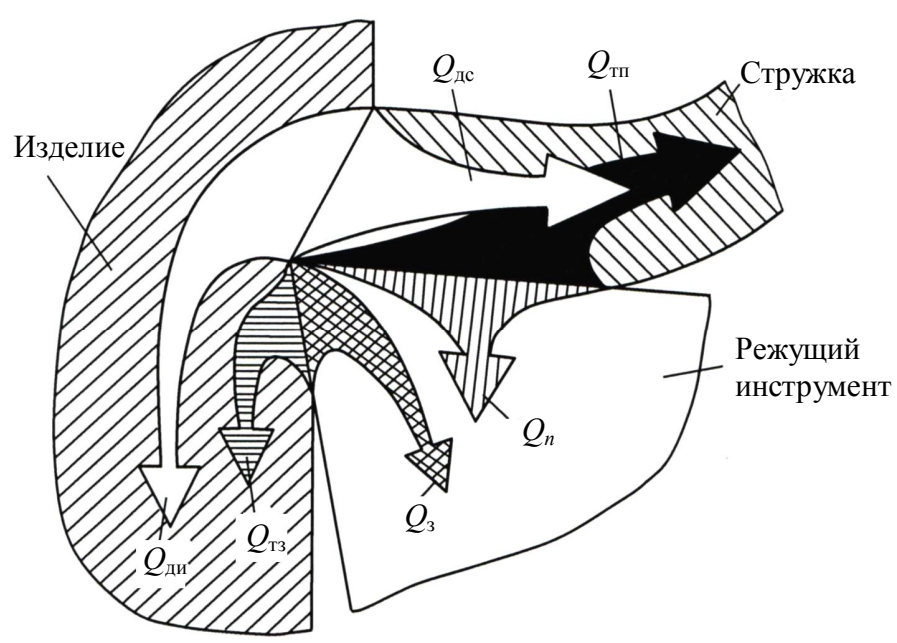

Рис.1. Количество тепла в изделии $Q_{\text {и инструмента } Q_{\mathrm{p}}}$ и стружке $Q_{\mathrm{c}}$ 
тате теплообмена на площадке контакта передней поверхности инструмента со стружкой; $Q_{\text {тп }}-$ часть теплоты, возникающая в результате трения между резцом и стружкой.

Содержание исследования. Определение температуры в поверхностном слое от каждого объемного источника тепла (рис.2) связано с интегрированием следующих выражений [1]:

$$
\begin{gathered}
\theta_{1}=\frac{q_{1} \exp (-p)}{2 \sqrt{\pi \lambda c \rho v}} \int_{\Delta_{1}}^{\Delta_{1}+\Delta_{2}} \exp \left(p \frac{x_{1}}{x_{2}}\right) \frac{d x_{1}}{\sqrt{x-x_{2}}} \int_{0}^{a_{1}} \exp \left[-\frac{p \operatorname{ctg} \beta_{1} y_{1}}{\Delta_{2}}-\frac{v\left(y-y_{1}\right)^{2}}{4 a\left(x-x_{1}\right)}\right] d y_{1} ; \\
\theta_{2}=\frac{q_{2} \exp (-p)}{2 \sqrt{\pi \lambda c \rho v}} \int_{0}^{\Delta_{2}} \exp \left(p \frac{x_{2}}{\Delta_{2}}\right) \frac{d x_{1}}{\sqrt{x-x_{2}}} \int_{0}^{h} \exp \left[-v \frac{\left(y-y_{1}\right)^{2}}{4 a\left(x-x_{2}\right)}\right] d y_{2} ; \\
\theta_{3}=\frac{q_{3} \exp \left(1+\frac{\Delta_{2}}{\Delta}\right)_{\Delta_{2}+\Delta}}{2 \sqrt{\pi \lambda c \rho v}} \int_{\Delta_{2}} \frac{d x_{3}}{\sqrt{x-x_{2}}} \int_{0}^{h_{1}} \exp \left[-\frac{v\left(y-y_{3}\right)^{2}}{4 a\left(x-x_{3}\right)}\right] d y_{3}-\frac{q_{3} \exp \frac{1}{\Delta}}{2 \sqrt{\pi \lambda c \rho v}} \int_{\Delta_{2}}^{\Delta_{2}+\Delta} \frac{x_{3} d x_{3}}{\sqrt{x-x_{3}}} \int_{0}^{h_{1}} \exp \left[-\frac{v\left(y-y_{3}\right)^{2}}{4 a\left(x-x_{3}\right)}\right] d y_{3},
\end{gathered}
$$

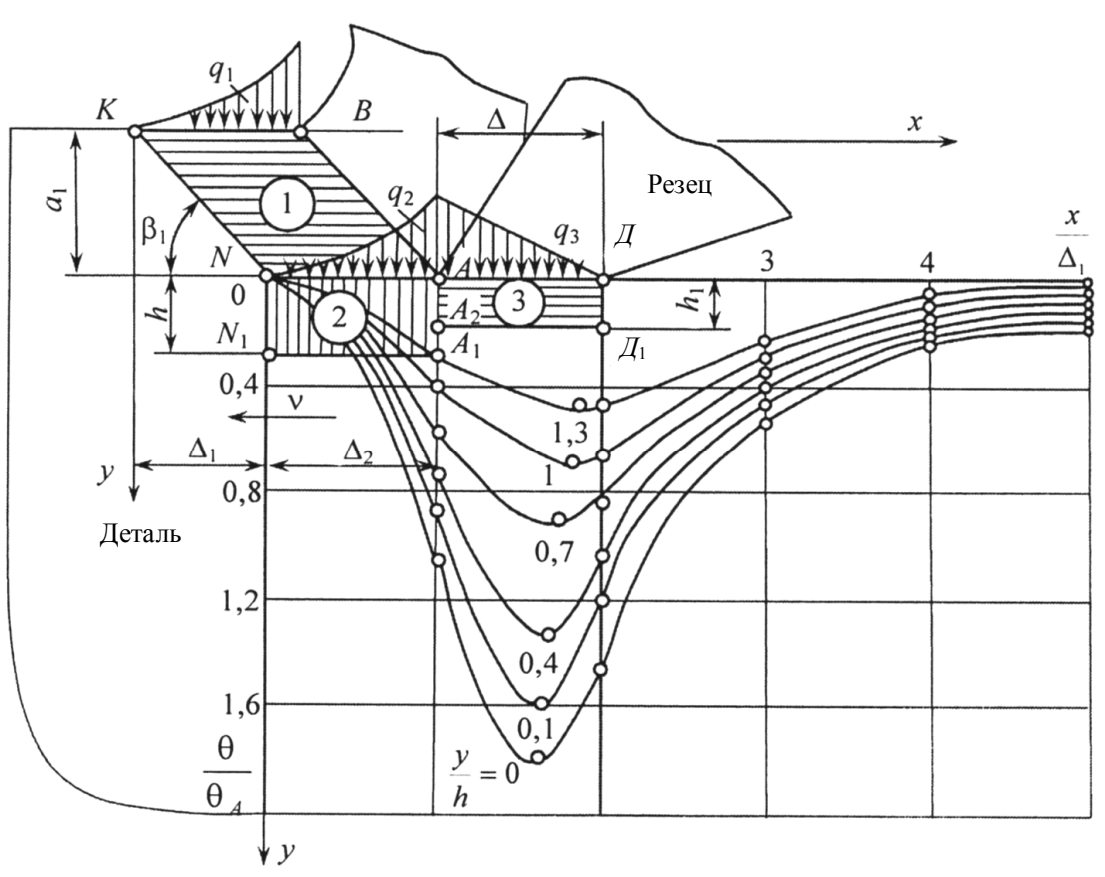

Рис.2. Схема действия объемных источников тепла и распределения температуры в поверхностном слое детали при обработке где $\theta_{1}, \theta_{2}, \theta_{3}-$ температура в поверхностном слое от первого, второго и третьего источников тепла соответственно; $v$ - скорость резания, м/с; $\lambda$ - теплопроводность материала обрабатываемой заготовки, Вт/(м.К); с $\rho$ - удельная объемная теплоемкость обрабатываемого материала, Дж/(м $\left.{ }^{3} \cdot \mathrm{K}\right) ; a$ - температуропроводность материала обрабатываемой заготовки, $\mathrm{m}^{2} / \mathrm{c}$; $a_{1}$ - толщина среза, м; $h$ - размер источника тепла $A N N_{1} A_{1} A$ вдоль оси $y, \mathrm{~m} ; h_{1}$ - размер источника тепла ДАА 2 Д Д вдоль оси $y, \mathrm{~m} ; p=5$ - константа, характеризующая распределение интенсивности тепловыделения; $\beta_{1}$ - угол наклона условной плоскости сдвига; $x_{1}$ и $y_{1}-$ ко-

ординаты линейного источника, м.

Исследования профессоров М.Ф.Полетики [4] и Н.В.Талантова [7] позволили А.Н.Резникову и С.С.Силину разработать расчетные схемы тепловых потоков в изделии, инструменте и стружке $[5,6]$, а также определить интенсивность их тепловыделения (рис.2).

Интенсивность тепловыделения при резании материалов в условной вершине режущего инструмента (точка $A$ на рис.2) определяется по формуле

$$
q_{A}=\tau_{\mathrm{p}} v \cos \beta_{1}
$$

где $q_{A}$ - интенсивность тепловыделения в условной вершине резца (в точке $\left.A\right)$, Дж/(м·c); $\tau_{\mathrm{p}}-$ сопротивление обрабатываемого материала пластическому сдвигу, Па; $\beta_{1}$ - угол наклона условной плоскости сдвига.

Интенсивность тепловыделения для первого, второго и третьего источников тепла (рис. 2) будет определяться по формулам:

$$
q_{1}=\frac{q_{A B} a_{1} \frac{1}{\sin \beta_{1}}}{\int_{0}^{\Delta_{2} h} \int_{0}^{h} \exp \left[-p\left(1-\frac{x_{1}-y_{1} \operatorname{ctg} \beta_{1}}{\Delta_{2}}\right)\right] d y_{1} d x_{1}} ;
$$




$$
\begin{gathered}
q_{2}=\frac{q_{A B} a_{1} \frac{1}{\sin \beta_{1}}}{\int_{0}^{\Delta_{2}} \int_{0}^{h} \exp \left[-p\left(1-\frac{x}{\Delta_{2}}\right)\right] \exp \left[-p \frac{y}{h}\right] d y_{2} d x_{2}} ; \\
q_{3}=\frac{p}{1-\exp (-p)} \frac{\tau_{p} v}{h_{1}} .
\end{gathered}
$$

На основе расчетов по формулам (1)-(3) и обработки результатов расчета получена упрощенная формула для определения температуры в поверхностном слое детали [1]:

$$
\left(\frac{\theta_{m}}{\theta_{A}}\right)_{O B}=R(B B)^{m-n_{y}}\left(\frac{h}{a_{1}}\right)^{n}\left(\frac{h_{1}}{h}\right)^{K}\left(\frac{\Delta}{\Delta_{1}}\right)^{P}\left(\frac{y}{h}\right)^{m_{y}}\left(\frac{\Delta_{1}}{\Delta_{2}}\right)^{U},
$$

где $\theta_{m}$ - температура в поверхностном слое детали на определенной глубине от поверхности, ${ }^{\circ} \mathrm{C}$; $\theta_{A}$ - температура в условной вершине режущей части инструмента (в точке $A$ ), ${ }^{\circ} \mathrm{C} ; y$ - глубина рассматриваемого слоя от поверхности, м; $\rho_{1}$ - радиус округления режущей кромки инструмента, м; $5=v a_{i} / a ; h=a_{1}\left(\sqrt{\frac{1}{B^{2}}-1}-1\right)$ - высота объемного источника тепла в зоне основных пластических деформаций (источника 2 ), м; $h_{1}=\Delta / \sqrt{2}$ - высота объемного источника тепла в зоне контакта режущего инструмента с обработанной поверхностью (источник 3 ), м; $\Delta=\arccos \left[1-a_{2} B^{-b_{2}(1-\sin \gamma)^{-x}}\right]+\frac{a_{2} B^{1-b_{2}(1-\sin \gamma)^{-x}}}{\sin \alpha(\cos \gamma+B \sin \gamma)}-$ протяженность теплового источника 3$), \mathrm{M} ; \gamma-$ передний угол режущей части инструмента, градус; $\Delta_{1}=a_{1} / B$ - проекция плоскости сдвига на направление перемещения режущего инструмента, м; $B=1 / \operatorname{tg} \beta_{1} ; \Delta_{2}=a_{1} / B \sqrt{2\left(1+B^{2}\right)}+1$ - протяженность теплового источника тепла $2, \mathrm{~m} ; R, m, n_{y}, k, p, m_{y}, u, a_{2}, x_{2}, b_{2}$ - зависят от свойств обрабатываемого и инструментального материала (табл.1, 2).

Температура резания в условной вершине резца (точка $A$, рис.2) определяется по формуле [6]

$$
\theta_{A}=\frac{\tau_{p}}{c \rho B} \operatorname{erf} \sqrt{\frac{E B}{4}} .
$$

Расчетом может быть определена скорость резания при лезвийной обработке и оптимальная скорость резания, соответствующая оптимальной температуре резания, при которой наблюдается минимум износа режущего инструмента. Последнее явление было открыто и обосновано профессором А.Д.Макаровым.

Оптимальная скорость резания определяется по формуле [6]

$$
\begin{gathered}
v_{0}=\frac{k^{2} \lambda c \rho \sin ^{0,2} \alpha \theta_{0}^{2} / \tau_{\rho}^{2}}{4 a_{1} c_{0}^{2}\left(\rho_{1} / a_{1}\right)^{2\left(n_{0}-0,1\right)}(1-0,45 \sin \gamma)\left(b / b_{1}\right)^{0,8}} \times \\
\times\left[1+\sqrt{1+\frac{2,65 \lambda_{p} \beta \varepsilon\left(a_{1} / b_{1}\right)^{0,3} c_{0}\left(\rho_{1} / a_{1}\right)^{2\left(n_{0}-0,1\right)}(1-0,45 \sin \gamma)\left(b / b_{1}\right)^{0,8} \tau_{\rho}}{k^{2} \lambda c \rho \sin ^{0,2} \alpha \theta_{0}}}\right]^{2}
\end{gathered}
$$

где $b$ - длина контакта режущей кромки инструмента с обрабатываемым материалом, м; $c_{0}$ и $k-$ величины, зависящие от сочетаний технологических условий обработки; $\theta_{0}$ - оптимальная температура резания, ${ }^{\circ} \mathrm{C}$. 
Значения коэффициента и показателей степени

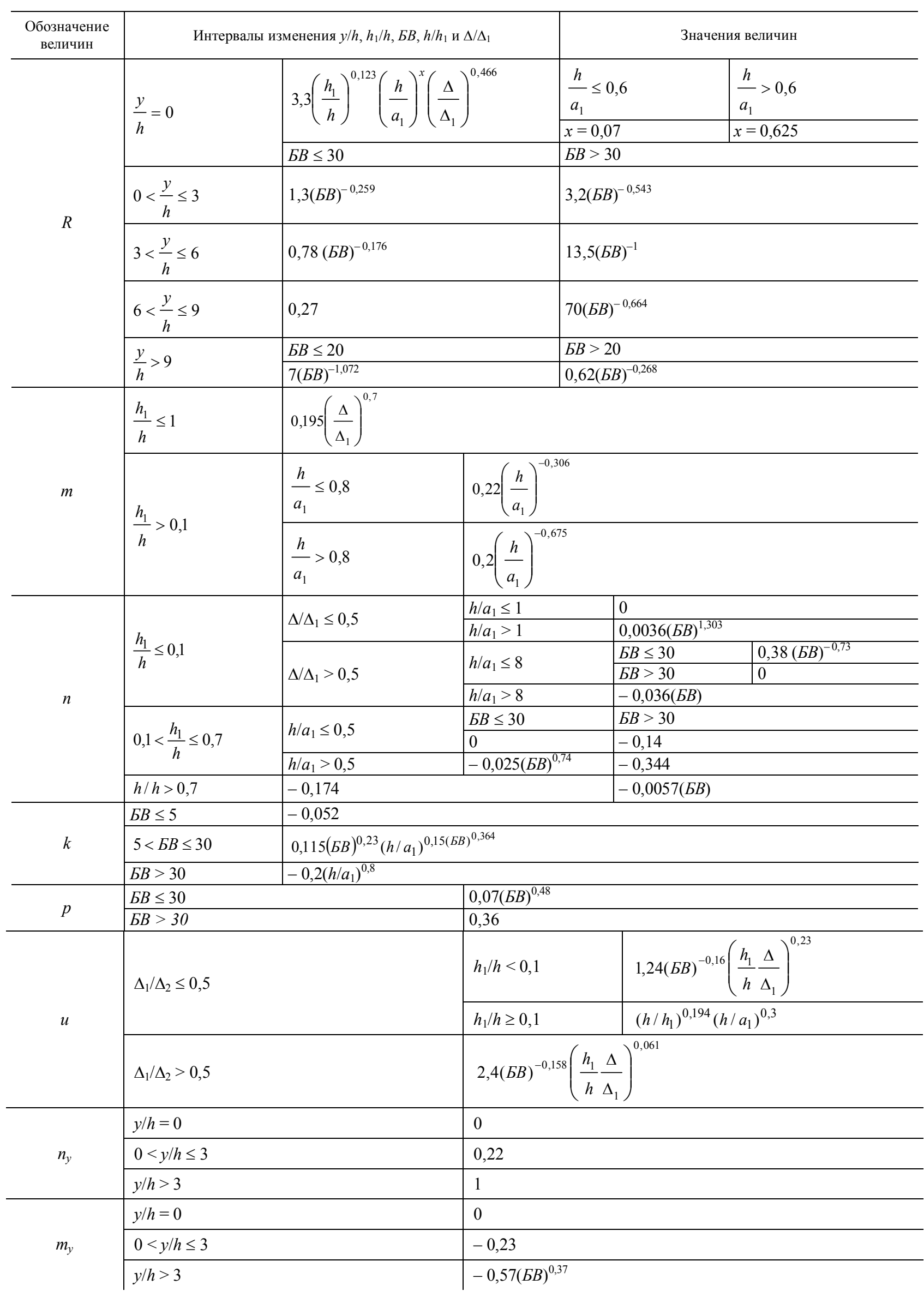


Таблича 2

Установлено, что при оптимальной температуре резания обеспечивается не только минимум износа режущего инструмента, но и наиболее благоприятные параметры качества поверхностного слоя [1].

Результаты исследований позволили с более высокой степенью точности выполнять расчеты погрешностей обработки, обусловленных тепловым воздействием на режущий инструмент и обрабатываемую заготовку.

Погрешность детали, обусловленная нагревом режущего инструмента в процессе обработки, определяется по формуле

$$
\begin{aligned}
& \Delta L_{\mathrm{p}}=\frac{\beta_{\text {ст }} \theta_{\mathrm{p}}}{2 K}\left\{\left(1+2 \sqrt{\frac{\alpha_{1} \alpha_{c m} \tau}{\pi \lambda_{c m} h}}\right) \sqrt{\frac{\lambda_{c m} h}{a_{1}}}\left[1-\exp \left(-L_{\mathrm{p}} \sqrt{\frac{\alpha_{1}}{\lambda_{c m} h}}\right)\right]-\right. \\
& -\frac{1}{\sqrt{\pi a_{c m} \tau}}\left[-\frac{\lambda_{c m} h}{\alpha_{1}} \exp \left(-L_{\mathrm{p}} \sqrt{\frac{\alpha_{1}}{\lambda_{c m} h}}\right)\left(L_{\mathrm{p}} \sqrt{\frac{\alpha_{1}}{\lambda_{c m} h}}+1\right)+\frac{\lambda_{c m} h}{\alpha_{1}}\right]- \\
& -\left(1-2 \sqrt{\frac{\alpha_{1} \alpha_{c m} \tau}{\pi \lambda_{c m} h}}\right) \sqrt{\frac{\lambda_{c m} h}{\alpha_{1}}}\left[1-\exp \left(-L_{\mathrm{p}} \sqrt{\frac{\alpha_{1}}{\lambda_{c m} h}}\right)\right]-\frac{1}{\sqrt{\pi \lambda_{c m} h}} \times \\
& \left.\quad \times\left[-\frac{\lambda_{c m} h}{\alpha_{1}} \exp \left(L_{\mathrm{p}} \sqrt{\frac{\alpha_{1}}{\lambda_{c m} h}}\right)\left(L_{\mathrm{p}} \sqrt{\frac{\alpha_{1}}{\lambda_{c m} h}}-1\right)+\frac{\lambda_{c m} h}{\alpha_{1}}\right]\right),
\end{aligned}
$$

где $\beta_{\mathrm{p}}$ - коэффициент температурного линейного расширения материала державки резца, $1 /{ }^{\circ} \mathrm{C} ; L_{\mathrm{p}}-$ длина вылета резца, м; $\theta_{\mathrm{p}}$ - температура в зоне резания, ${ }^{\circ} \mathrm{C} ; K=\pi^{2} / 2 \beta \varepsilon$ - величина, учитывающая геометрию инструмента при несвободном резании; $\alpha_{1}$ - коэффициент теплоотдачи материала державки резца в окружающую среду, Дж/( $\left.\mathrm{M}^{2} \cdot \mathrm{c} \cdot{ }^{\circ} \mathrm{C}\right) ; a_{c m}$ и $\lambda_{c m}-$ коэффициенты температуропроводности и теплопроводности материала державки резца, м/ $\mathrm{c}^{2}, \mathrm{BT} /(\mathrm{M} \cdot \mathrm{K}) ; h$ - отношение площади сечения державки резца к его периметру, м; $\tau$ - время работы резца, с; $\beta$ и $\varepsilon-$ угол заострения и угол при вершине режущей части инструмента в плане, рад.

Значение $\theta_{\mathrm{p}}$ определяется по формуле [2]

$$
\begin{aligned}
\theta_{\mathrm{p}}=\frac{2 \theta_{N} \sqrt{a_{c m} \tau}}{H\left[-E_{i}\left(-\frac{\Delta^{2}}{16 a_{c m} \tau}\right)\right]}\left\{\frac{H}{2 \sqrt{a_{c m} \tau}}\left[-E_{i}\left(-\frac{H^{2}}{4 \sqrt{a_{c m} \tau}}\right)\right]-\frac{\Delta}{4 \sqrt{a_{c m} \tau}}\left[-E_{i}\left(-\frac{\Delta^{2}}{16 a_{c m} \tau}\right)\right]+\right. \\
\left.+2 \pi\left(\operatorname{erf} \frac{H}{2 \sqrt{a_{c m} \tau}}-\operatorname{erf} \frac{\Delta}{4 \sqrt{a_{c m} \tau}}\right)\right\},
\end{aligned}
$$

где $H$ - высота державки резца; $E_{i}$ - интегрально показательная функция Эйлера; $e r f$ - интеграл вероятностей; $\theta_{N}-$ максимальная температура на задней поверхности резца, определяемая по формуле С.С.Силина [6].

Температура в середине площадки контакта режущего инструмента с обрабатываемой заготовкой определяется по формуле [5]

$$
\theta_{N}=\theta_{A}\left(0,5+\frac{0,36 \sin ^{0,25} \alpha}{B^{1,25} \sqrt{5 E}}+\frac{0,465 B^{1,275} D^{0,625} E^{0,55} \cos \alpha}{\Gamma^{0,25} Д^{0,075} \sin ^{0,275} \alpha \operatorname{erf} \sqrt{\frac{5 B}{4}}}\right),
$$


где $E=\rho_{1} / a_{1}-$ безразмерный комплекс, характеризующий влияние на процесс обработки геометрической формы режущей кромки резца; $Д=a_{1} / b_{1}-$ безразмерный комплекс, характеризующий геометрию сечения резца; $\Gamma=\left(\lambda_{\mathrm{p}} / \lambda_{\text {д }}\right) \beta \varepsilon$ - безразмерный комплекс процесса резания, характеризующий влияние геометрии режущей части инструмента и отношение теплопроводности обрабатываемого и инструментального $\lambda_{\text {p }}$ материалов; $\beta$ и $\varepsilon-$ угол заострения режущей кромки инструмента и угол при вершине резца в плане, радиан.

Погрешность обработки, обусловленная температурой деформацией обрабатываемой детали, определяется по формуле:

$$
\Delta R=\frac{C \beta_{\text {д }} \theta_{\text {max }} \mathrm{Fo}^{m}}{0,5 R_{\mathrm{H}}+1}\left(\frac{l}{S_{\mathrm{M}}}\right)^{n}\left(R_{\mathrm{H}}-\left(\frac{R_{\mathrm{B}}}{R_{\mathrm{H}}}\right)^{0,5 R_{\mathrm{H}}} R_{\mathrm{B}}\right),
$$

где $\beta_{\text {д }}$ - коэффициент температурного линейного расширения материала обрабатываемой детали, $1 /{ }^{\circ} \mathrm{C} ; R_{\mathrm{H}}$ и $R_{\mathrm{B}}-$ наружный и внутренний радиусы обрабатываемых поверхностей, м; $S_{\mathrm{M}}-$ минутная подача режущего инструмента, м; $l$ - длина теплового источника, движущегося по обрабатываемой поверхности, м; Fo $=a \tau / R_{\mathrm{H}}-$ критерий Фурье; $\tau-$ время нагревания, ${ }^{\circ} \mathrm{C} ; C, m$ и $n-$ величины, зависящие от значений минутной подачи режущего инструмента и критерия Фурье [1].

Заключение. Полученные расчетные зависимости для определения температуры в зоне резания и погрешностей обработки, обусловленных тепловым воздействием на обрабатываемую заготовку и режущий инструмент, позволяют прогнозировать их величину при технологической подготовке производства на основе назначения режимов резания и выбора марки режущей части инструмента и ее геометрических параметров.

\section{ЛИТЕРАТУРА}

1. Безъязычный В.Ф. Метод подобия в технологии машиностроения. М.: Машиностроение, 2012. 320 с.

2. Красный B.A. Триботехнические характеристики деталей горных машин с регулярной микрогеометрической поверхности / В.А.Красный, В.В.Максаров // Металлообработка. 2016. № 1(91). С. 29-35.

3. Максаров В.B. Моделирование и управление динамическими свойствами технологических систем / В.В.Максаров, П.В.Леонидов // Записки Горного института. 2014. Т. 209. С. 71-77.

4. Полетика М.Ф. Теория резания. Ч. 1. Механика процесса резания. Томск: Изд-во Томского политехнического университета. 2001. $202 \mathrm{c}$.

5. Резников А.Н. Теплофизика процессов механической обработки материалов. М.: Машиностроение, 1981. 279 с.

6. Силин С.С. Метод подобия при резании материалов. М.: Машиностроение, 1979. 152 с.

7. Талантов Н.В. Физические основы процесса резания, изнашивания и разрушения инструмента. М.: Машиностроение, 1992. 240 c.

8. Boothroyd G. Temperatures in Orthogonal Metal Cutting // Proc. Jnst. Mech. Eng. 1963. Vol. 177. P. 789-810.

9. Maksarov V.V. Increasing the Effectiveness of the Cutting Process in the Course of Milling / V.V.Maksarov, Ju.Ju.Olt, M.M.Madissoo // Journal of Mechanics \& Industry Research. 2013. Vol. 4. № 1. P. 75-81.

10. Maksarov V.V. Increase of wear resistance of friction down hole oil pumps with seals of directionally reinforced polymer composizioni materials / V.V.Maksarov, V.A.Krasnyy // Chemical and Petroleum Engineering. 2017. Vol. 1. P. 34-37.

11. Mathematical Modelling of Cutting Process System / Ju.Ju.Olt, O.O.Liivapuu, V.V.Maksarov, A.A.Liyvapuu, T.T.Tärgla // Engineering, Mathematics I. Springer. Proceedings in Mathematics \& Statistics. 2016. Vol. 178. P. 173-186.

Aвторы: В.Ф.Безъязычный, д-р техн. наук, професcop, tehnology@rsatu.ru (Рыбинский государственный авиационный технологический университет имени П.А.Соловьева, Рыбинск, Россия), М.Счерек, д-р техн. наук, профессор, заместитель директора по науке и исследованиям, marian.szcerek@gmail.com (Институт экологически рачиональных технологий Национального научно-исследовательского института, Радом, Польша).

Статья поступила в редакиию 7.02.2018.

Статья принята к публикации 6.04.2018. 